\title{
Acoustics Based Monitoring and Diagnostics for the Progressive Deterioration of Helical Gearboxes
}

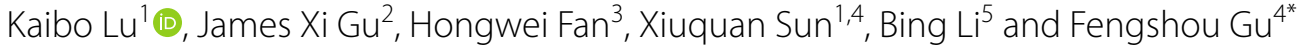

\begin{abstract}
Gearbox condition monitoring (CM) plays a significant role in ensuring the operational reliability and efficiency of a wide range of critical industrial systems such as wind turbines and helicopters. Accurate and timely diagnosis of gear faults will improve the maintenance of gearboxes operating under sub-optimal conditions, avoid excessive energy consumption and prevent avoidable damages to systems. This study focuses on developing CM for a multi-stage helical gearbox using airborne sound. Based on signal phase alignments, Modulation Signal Bispectrum (MSB) analysis allows random noise and interrupting events in sound signals to be suppressed greatly and obtains nonlinear modulation features in association with gear dynamics. MSB coherence is evaluated for selecting the reliable bi-spectral peaks for indication of gear deterioration. A run-to-failure test of two industrial gearboxes was tested under various loading conditions. Two omnidirectional microphones were fixed near the gearboxes to sense acoustic information during operation. It has been shown that compared against vibration based CM, acoustics can perceive the responses of vibration in a larger areas and contains more comprehensive and stable information related to gear dynamics variation due to wear. Also, the MSB magnitude peaks at the first three harmonic components of gear mesh and rotation components are demonstrated to be sufficient in characterizing the gradual deterioration of gear transmission. Consequently, the combining of MSB peaks with baseline normalization yields more accurate monitoring trends and diagnostics, allowing the gradual deterioration process and gear wear location to be represented more consistently.
\end{abstract}

Keywords: Acoustics, Modulation Signal Bispectrum, Helical gearbox, Gear wear

\section{Introduction}

In industrial applications, gearboxes are widely used for transmitting power from one mechanical system to another. Gear wear, the progressive material loss from contacting tooth surfaces due to a combination of rolling and sliding motion under mixed or boundary lubrication conditions, is one of the major faults of gears. This directly results in strong vibration and noise, dynamic transmission error, and power transmission deficiency [1-3]. For modern machinery the development and the

\footnotetext{
*Correspondence: f.gu@hud.ac.uk

${ }^{4}$ Centre for Efficiency and Performance Engineering, University

of Huddersfield, Huddersfield HD1 3DH, UK

Full list of author information is available at the end of the article
}

parallel requirements for prolonged life-cycle, improved production quality, enhanced human safety and reduced adverse environmental impacts, have led to increasing motivation for more effective maintenance strategies.

Modern condition monitoring (CM) techniques employ advanced technologies to evaluate the machine health and predict when and where the machine is likely to fail, thereby maximizing the machine profitability. Generally, vibration sensors are adopted for $\mathrm{CM}$ of rotating machinery [2-4] or manufacturing processes $[5,6]$. It is generally accepted that vibration signals from a gearbox depend on the sensor location relative to the source and are often contaminated by structure-borne noises from other sources, leading to a low signal noise ratio (SNR). To increase the SNR, researchers $[7,8]$ have used the technique of the time 
synchronous averaging (TSA) aided with instantaneous angular speed signals in detecting missing teeth and general gear wear in the multistage gearboxes.

During the past few years, acoustics-based CM of machines has been a popular subject of study amongst scholars [9-15]. Acoustics-based CM techniques are generally non-intrusive, inexpensive and easily-mountable. It is well-known that acoustics as well as vibrations will be inevitably generated when the machine is in operation $[9,10]$. These acoustic signals can be picked up using special microphones and processed by appropriate techniques to extract useful information on the health condition of the machine. Acoustics-based techniques have gradually been regarded as an alternative option for CM of bearings, gearboxes and engines.

$\mathrm{Gu}$ et al. [11] developed an acoustic monitoring method to detect tappet clicks, misfiring and injection timing faults in diesel engines with the use of a discrete wavelet transform signal processing method. Following this pioneering work, Ball et al. [12] evaluated the feasibility of using acoustics for fault detection in electrical motors with the discrete wavelet transform and averaging techniques. The results showed that the acoustics-based method is effective in extracting fault information, even in industrial environments where the background noises are relatively strong. Henceforth, other advanced techniques for signal processing including the continuous wavelet transform and independent component analysis, were also applied to the acoustical signals from engines [13, 14]. Jiang et al. [15] proposed a distinctive $\mathrm{CM}$ method for diesel combustion engines based on the acoustic source characterization of the exhaust system. Zhou et al. [16] introduced a comprehensive investigation into the noise radiation and dynamic response of a gearbox using the finite element simulation method. For quality control of machining processes, scholars [17-19] also measured the audible sound emitted in operation to monitor self-excited vibrations or the tool wear during cutting.

Acoustic monitoring can provide a direct indication of noise sources in a machine. However, a major issue with this method is the background noise contamination, especially the noise coming from the connected sound sources, for example, cooling fans, drive motor and/or a nearby machinery. Bispectrum is a statistic calculation applied to search for the nonlinear interaction of continuous spectra [20]. Bispectrum analysis has a number of distinctive advantages when compared to the traditional power spectrum analysis. These include preservation of phase information, Gaussian noise elimination and nonlinear component (modulation) recognition $[21,22]$.
In this study, an evaluation of the CM performance of a two-stage helical gearbox using the airborne acoustics, in conjunction with an updated Modulation Signal Bispectrum (MSB) analysis, is performed. The acoustic signals can be obtained remotely and conveniently from the gearbox under investigation. First the theoretical backgrounds of gear dynamics MSB are introduced. Thereafter, through run-to-failure experimentations of the gearbox test rig, the proposed CM method is evaluated and compared with the conventional vibration-based method. Finally, conclusions of this study are given.

\section{Theoretical Backgrounds}

\subsection{Dynamics of Gear Transmissions}

In a gear transmission system, the gear pair assembly remains one of the major sources of vibration and noise. The vibration of the gears is generated due to the fluctuation of the dynamic meshing force affected by the timevarying mesh stiffness and gear errors [23]. The dynamic model of an engaged gear pair can be expressed as [24]

$$
M \ddot{x}+C \dot{x}+k(t) x=k(t) E_{1}+k(t) E_{2}(t),
$$

where $M, C$ are the equivalent mass and damping of the gear pair, respectively, $E_{1}$ is the average static elastic deformation of the gear teeth under loading, $E_{2}(t)$ is the relative displacement between the meshing gears associated with gear faults as well as manufacturing and installing errors, and $k(t)$ is the time-varying mesh stiffness related to the gear profile and is a periodic function with the period of the gear mesh. Even a small amount of wear has a significant effect on the profile of the mesh stiffness $[25,26]$. As a result, the gear pair will deviate from the tooth profile in reference to its ideal involute and result in high vibration and acoustics.

Representing the periodic function in terms of Fourier series in Eq. (1) it can be documented that the gear mesh frequency (GMF) and its harmonics as the key components of the dynamic response of a gear transmission system are always modulated by the shaft/gear imperfection, such as unbalance, misalignment, gear wear, pitch errors and so on [24-28]. Thus, GMFs and their associated sidebands which can be characterized in Eq. (2), are always dominantly observable due the nonlinearity of vibration responses.

$$
f=n f_{m} \pm k f_{r},
$$

where $f_{m}$ is the GMF, $f_{r}$ is the shaft rotational frequency (SRF), $n=0,1,2, \ldots$ and $k=0,1,2, \ldots$, etc. Due to wear on the tooth profiles the coupling of these frequencies and their respective amplitudes will change with operating time. Technically, any such components at $n f_{m} \pm k f_{r}$ 
can be used for indicating the gradual deteriorations of gears such as surface wear and tooth cracks.

\subsection{Acoustic Responses to Gear Wear}

Tooth wear alters the mesh stiffness, thus varies the parametric excited vibrations of the gear set during gear meshing. The excitation propagates from the mated gears to the shafts and bearings and cause the housing of the gearbox to vibrate. The surface vibration of the gearbox causes fluctuations of the air surrounding the gearbox housing, generating airborne sound. These air fluctuations then formulate sound waves that can propagate far away. This means that the airborne sound and the structural vibration are sourced from the same excitations, with different transmission media and paths.

Moreover, the sound signal perceived at a position in the direct sound field is the superposition of the sounds radiated from different parts of a vibration object. This means that when compared with the vibration measured on a local part of the object, acoustic sound can more comprehensively or globally reflect the dynamics of the object.

In addition, acoustic sensing also offers the benefit that the $\mathrm{CM}$ of a gearbox can be achieved by remote measurements of sound waves. This provides great convenience for its implementation in many industrial applications, where traditional vibration or oil detection sensors are prohibitively difficult to attach to the gearboxes, such as in the mining industry. However, obtaining accurate and reliable diagnostic information from the noisy acoustic measurements is more challenging since acoustic signals are influenced by both background noises and the acoustics of nearby sources.

\subsection{MSB Analysis}

The acoustic signals consist of distinctive modulation characteristics, MSB analysis is selected as the promising tool to suppress noise influences and extract the modulation characteristics. MSB takes into account the property of phase invariance of the modulation signals which allows more accurate and robust modulation estimation by the suppression of the influences of random noises. Studies in Refs. [29-31] have shown that MSB allows accurately extracted modulation characteristics from motor current signals and achieves outstanding performance in monitoring the gradual deteriorations of gearboxes. MSB implementation does not need additional phase alignment signals which time synchronous averaging (TSA) requires, therefore allowing for less complex and more cost-effective practical CM strategies to be implemented.
According to MSB definition in the frequency domain, the mesh frequency component $f_{m}$ and its sideband $f_{m} \pm f_{r}$ at in a signal can be correlated [20-22] as

$$
B_{M S}\left(f_{m}, f_{r}\right)=E\left[X\left(f_{m}+f_{r}\right) X\left(f_{m}-f_{r}\right) X^{*}\left(f_{m}\right) X^{*}\left(f_{m}\right)\right],
$$

where $E[]$ is the statistical expectation operator, $X^{*}(f)$ is the complex conjugate of Fourier transform $X(f)$ of the calculated signal $x(t)$. At $f_{r}=0$ Eq. (3) can result in the power spectrum of $x(t)$ :

$$
P\left(f_{m}\right)=E\left[X\left(f_{m}\right) X^{*}\left(f_{m}\right)\right] .
$$

Through the complex averaging process in the frequency domain as shown in Eq. (3), the product of MSB aligns the time invariant phases of modulation sidebands which preserves the modulation content such that of the mesh frequency $f_{m}$, the higher sideband $f_{m}+f_{r}$ and the lower sideband $f_{m}-f_{r}$. On the other hand, the nonmodulation components and random noises can be significantly suppressed because they do not meet the phase invariance relationship. Thus, the effect of the modulation in the signal can be represented more reliably and accurately.

Furthermore, MSB coherence (MSB-coh) or normalized MSB can be defined as

$$
B_{M S}^{2}\left(f_{m}, f_{r}\right)=\frac{\left|B_{M S}^{2}\left(f_{r}, f_{m}\right)\right|^{2}}{E\left[\left|X\left(f_{m}\right) X^{*}\left(f_{m}\right)\right|^{2}\right] E\left[\left|X\left(f_{m}+f_{r}\right) X\left(f_{m}-f_{r}\right)\right|^{2}\right]} .
$$

MSB-coh is valued with a boundary of $[0,1]$. The value of one indicates that the magnitude of MSB is absolutely induced by the modulation effect. On the other hand, the value of zero means that the magnitude of MSB is primarily from the influence of random noises or no modulation effect. Therefore, MSB-coh values can assist to evaluate the reliability of the bi-spectral peaks in Eq. (3), which will be helpful in selecting MSB peaks for reliable indication for dynamic effects. In addition, with the bounded values, MSB-coh can be presented graphically for easily detecting modulation components with smaller amplitude in a multiple complicated signals, which is particularly useful for acoustic signal analysis as it often has many tens of decibels $(\mathrm{dB})$ in amplitude differences, which is difficult to be presented by MSB magnitudes. However, as MSB-coh amplitudes are influenced by noise it is not so suitable to indicate the degrees of modulation as that of noise purified MSB magnitudes.

In addition, MSB-coh can be also based to verify MSB calculation settings and data stationarity. If its value shows a monotonic decrease with averaging process or the expectation, it indicates that the signal is stationary or has less interrupting events. At the end of the 
averaging process, the value should be stable, otherwise the signal is too noisy and more data is required for the expectation.

\section{Experimentation}

\subsection{Test Rig}

A run-to-failure test, which took more than $800 \mathrm{~h}$ totally, was performed on a two-stage helical gear system to verify the capability of acoustic signal based gear condition monitoring approach. The experimental setup is shown in Figure 1. The test rig of the gearbox consists of an AC motor as the driving power, two helical back-to-back gearboxes as the testing objects, and a DC generator as the load device. The components are linked by the flexible couplings. Both motors are operated by a PLC system to control the rig at various specified load and speeds automatically. The gearboxes were supplied from a major gearbox company, consisting of two stage helical gears with a rated capacity of $10 \mathrm{~kW}$ and are widely used in different industries. The industrial grade gearboxes generate more informative data with greater generic characteristics that make the data analysis technique be evaluated fully.

Figure 2 provides the schematic diagram of the gear transmission system. The two gearboxes have the same transmission ratio $Z_{r 3} / Z_{r 4}=Z_{i 2} / Z_{i 1}=13 / 59$ at the lower speed stages but they have a different number of gear teeth at the higher speed stages. Gearbox 1, denoted as GB1, operating as a speed reducer has the gear ratio of $Z_{r 1} / Z_{r 2}=49 / 55$, whereas gearbox 2 , denoted as GB2, operating as a speed increaser has the ratio of $Z_{i 3} / Z_{i 4}=47 / 58$. These configurations make the DC generator operates with high efficiency and applies sufficient loads to the system over a wide range of speeds.

The symbols $f_{r}$ and $f_{m}$ represent the shaft rotational frequency (SRF) and the gear mesh frequency (GMF), respectively. In addition, the numerical subscripts are assigned for different gear shaft and gear mesh pairs as illustrated in Figure 2. Table 1 gives these frequency values at the AC motor speed of $f_{r 1}=17.3 \mathrm{~Hz}$.

During experiments, two CHZ-211 omnidirectional microphones with sensitivity of $48 \mathrm{mV} / \mathrm{Pa}$ were respectively fixed at a distance of approximately $350 \mathrm{~mm}$ above both gearboxes, as seen in Figure 1. This microphone installation is closer to the gearboxes but further away from the motors, which senses more acoustic signals in the stable sound field formulated from the tested gearboxes. Both microphones are also less affected by background sound sources such as the driving AC motor and the loading generator, as well as reflections from the shop floor and ceilings.

Table 1 Gearbox characteristic frequencies

\begin{tabular}{lll}
\hline Gearbox No. & SRF $(\mathbf{H z})$ & GMF (Hz) \\
\hline GB1 & $f_{r 1}=17.3$ & $f_{m 1}=847.7$ \\
& $f_{r 2}=15.4$ & \\
& $f_{r 2}=15.4$ & $f_{m 2}=200.2$ \\
& $f_{r 3}=3.39$ & \\
GB2 & $f_{r 3}=3.39$ & $f_{m 3}=200.2$ \\
& $f_{r 4}=15.4$ & \\
& $f_{r 4}=15.4$ & $f_{m 4}=723.84$ \\
& $f_{r 5}=12.48$ & \\
& &
\end{tabular}

Figure 1 Test system set-up

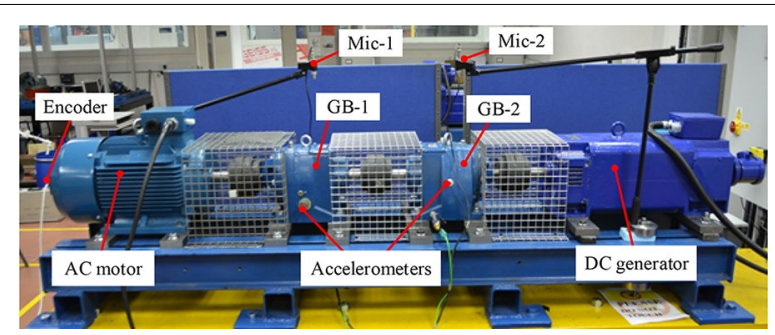

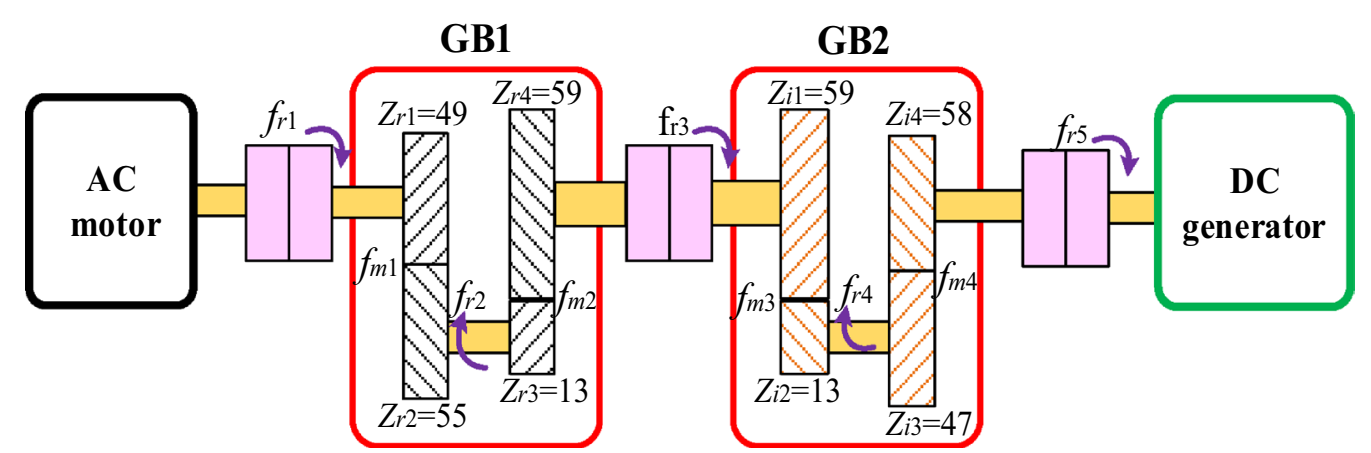

Figure 2 Schematic diagram of back-to-back gearboxes 
In addition, two YMC121A20 accelerometers with sensitivity of $20 \mathrm{mV} / \mathrm{g}$ were attached on the housings of the two gearboxes separately to measure the surface vibrations, and an optical encoder for TSA analysis was fixed at the non-driving shaft end of the AC motor, as shown in Figure 1.

\subsection{Run-to-Failure Test Implementation}

During the course of the test, the gearboxes were operating under various loading conditions to simulate the gear deterioration under its natural operating conditions as closely as possible. The loading types include oscillating loads, shock loads, and a range of constant loads at a wide range of speeds, which are similar to the practical scenarios of many gearboxes equipped in helicopters and wind turbines.

The variable conditions for one operation cycle lasted $60 \mathrm{~min}$, as illustrated in Figure 3. This was programmed into the PLC and repeated throughout the entire test procedure. These variable loads and speeds were designed to vary the level of both the steady and dynamic loads acting on the gears, and hence changing fatigue stress, deformation of the teeth and lubrication regimes. The motor speed was around $1040 \mathrm{r} / \mathrm{min}$ or $17.3 \mathrm{~Hz}$ during operation. The sample rate was set to be $96 \mathrm{kHz}$. For each loading phase, all signals were recorded continuously for $30 \mathrm{~s}$ and then processed off line using the proposed method. The setting enables the spectrum resolution to be sufficiently high so that the sidebands can be resolved accurately. In particular, the long data record ensures sufficient averages to be achieved in MSB calculation for reliable monitoring results.

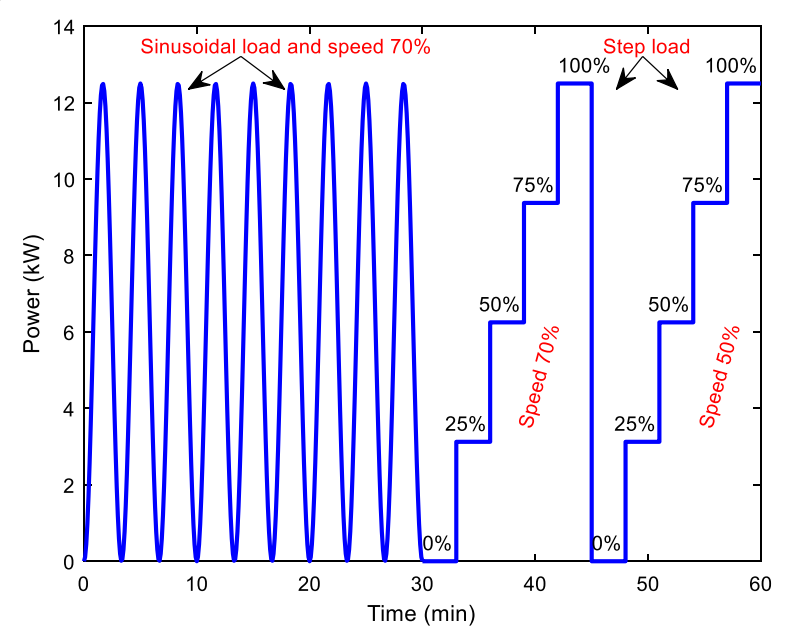

Figure 3 Load profile for a test operation cycle
Moreover, during the test course, no other refining actions such as changing lubricants, realigning couplings, and so on were carried out in order to maintain consistency of gear mesh dynamics and obtain reliable and consistent monitoring datasets. The run-to-failure test was terminated when it was found that there was a threefold increase in either acoustic or vibration monitoring features.

\section{Results and Discussion}

\subsection{Baseline Acoustic Signatures}

To confirm that the acoustic measurements contain sufficient information about dynamics of the gears, the spectra of the acoustic signals were compared with that of the vibration signals. Figure 4 presents a typical comparison result at the early stage of the operation when the running-in period of around $325 \mathrm{~h}$ is completed. As seen in Figure 4, the acoustic spectra display more components, including the mesh frequencies of $f_{m 1}, f_{m 2}, f_{m 3}, f_{m 4}$ and their harmonics and sidebands with significant amplitudes. This shows consistency with the vibration spectra. Therefore, it is proven that the acoustic signals measured in such a remote way are adequate and suitable for the condition monitoring of the gearboxes. Besides, because of the distinctiveness of these mesh related components it is a more reliable technique for diagnosing and tracking gear mesh conditions.

There also exist various inevitable noises, such as background noises, bearing and motor acoustic interferences, this simple spectrum analysis usually results in unsteady and unreliable results. Typically, spectral amplitudes will exhibit high fluctuations due to the contribution of additive stationary noises and the spectral leakages of other irrelevant significant components at, for example, $100 \mathrm{~Hz}, 550 \mathrm{~Hz}$ and $626 \mathrm{~Hz}$ which mostly originate from the $\mathrm{AC}$ motors, as highlighted by the red text in Figure 4. Moreover, the spectral amplitudes of interest at mesh and sideband frequencies can be also affected by nonstationary acoustic interruptions including transient operations of nearby machines and other activities in the manufacturing workshop. Therefore, it is necessary to use more advanced signal processing method such as MSB to attenuate such noises and obtain steady and reliable diagnostic features.

\subsection{Diagnostic Features}

To suppress the noise in acoustic signals, the MSB analysis was applied to the acoustic signal and yielded typical MSB results as shown by $3 \mathrm{D}$ mesh graphs of Figure 5 . Both MSB magnitude and coherence cover the low frequency range including several mesh and shaft frequencies of 


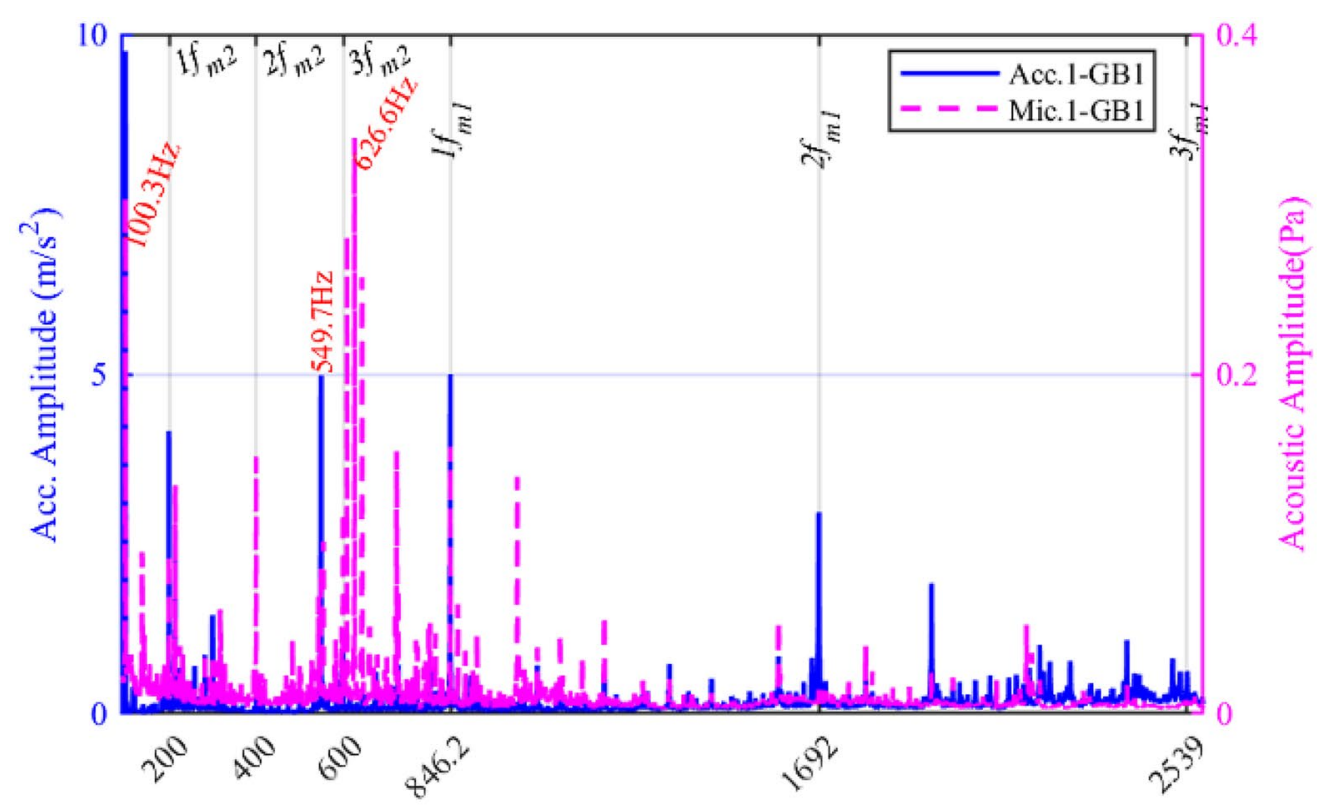

Frequency $(\mathrm{Hz})$

(a)

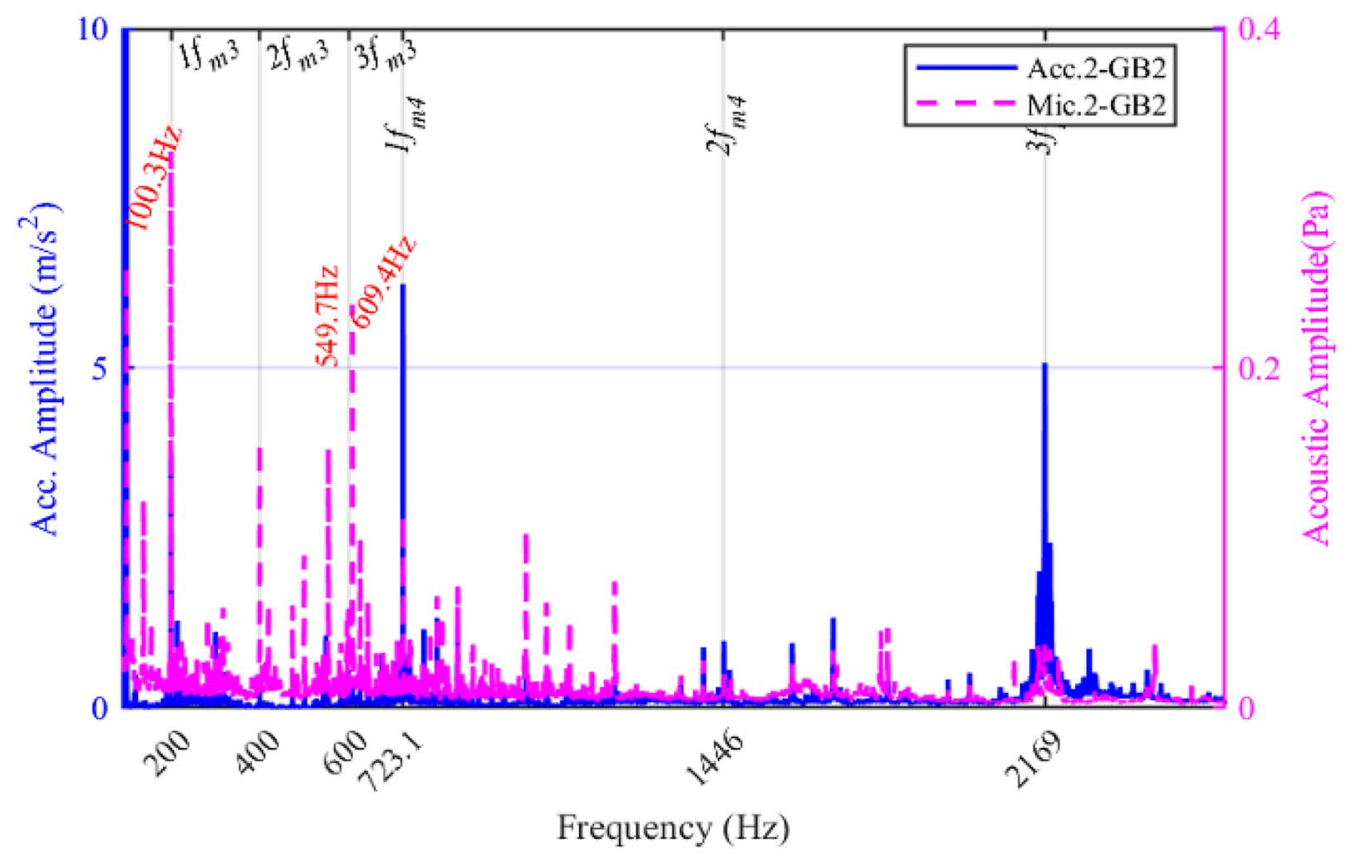

(b)

Figure 4 Spectral comparison between acoustic and vibration measurements: (a) Gearbox 1, (b) Gearbox 2 


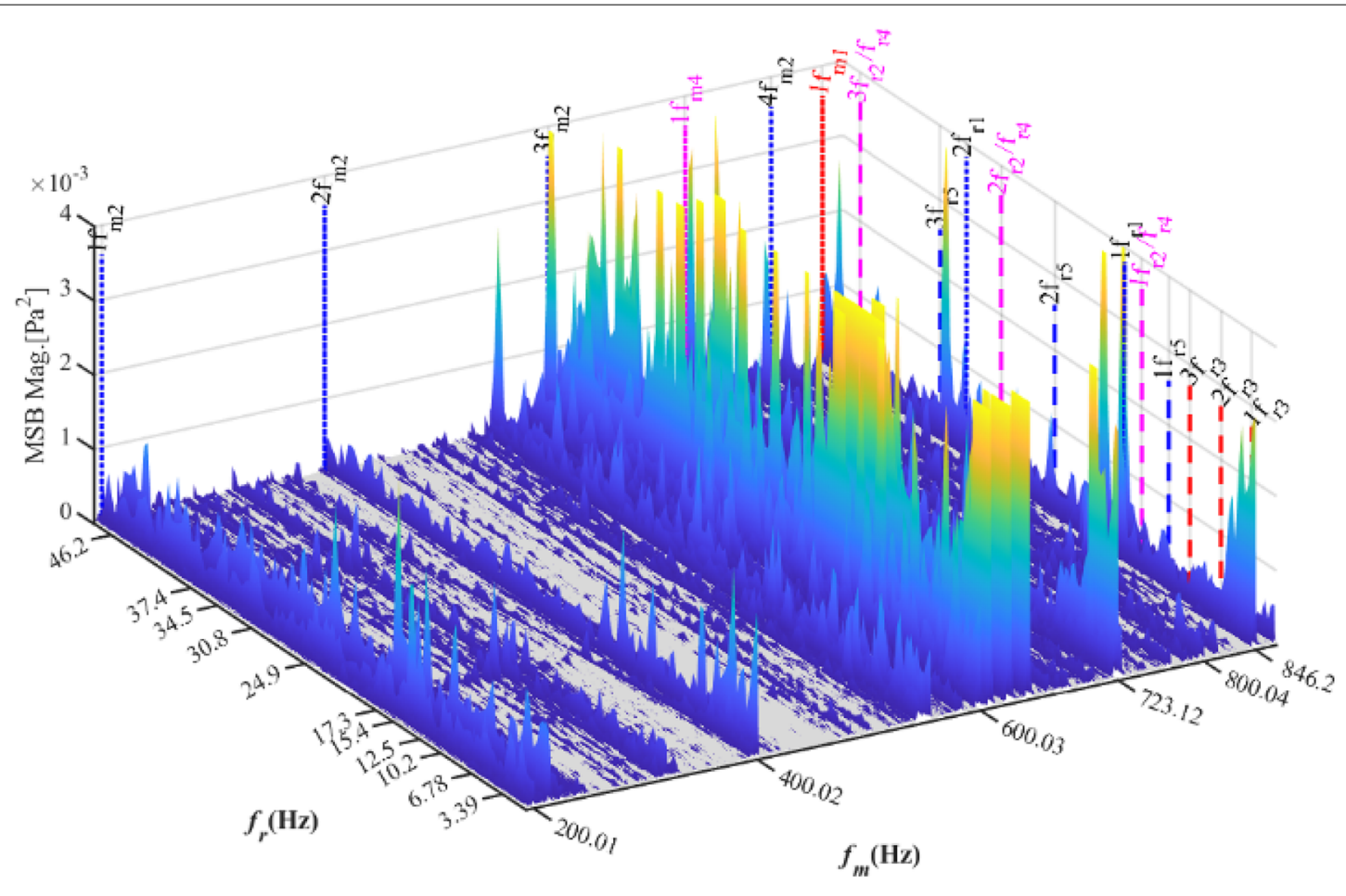

(a)

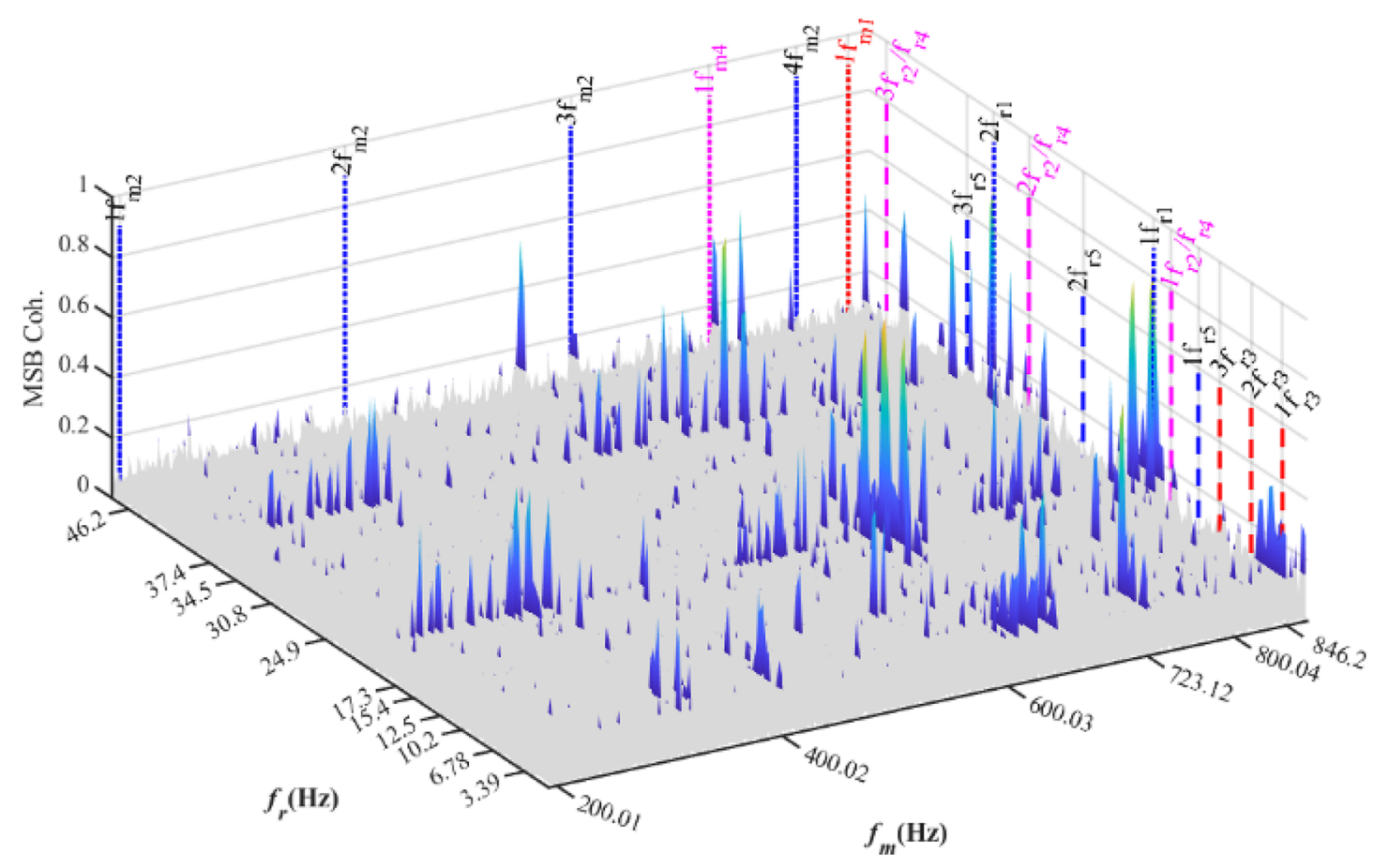

(b) 
interest. They were obtained by applying Eq. (3) to overlapped short signal segments and separating the long acoustic signals and averaging the complex MSBs of different segments. In total, 50 averages were achieved, at which the MSB coherence averaged for the bi-frequency regions $(0-60 \mathrm{~Hz}, 90-3000 \mathrm{~Hz})$ of interests becomes stable, indicating that the average is sufficiently good.

It can be seen from Figure 5(a) that MSB magnitude shows a large number of MSB peaks on the $f_{m}-f_{r}$ bifrequency plane. The most significant peaks do not appear at mesh frequencies, but at $f_{m}=626 \mathrm{~Hz}$, which is mainly from the AC motor as it is significantly coupled with motor speed of $f_{r 1}=17.3 \mathrm{~Hz}$ and $2 f_{r 1}=34.5$ Hz. This coupling can be more evidently observed by MSB coherence of Figure 5(b), in which the coherence peaks are appearing at $f_{r 1}=17.3 \mathrm{~Hz}$ and $2 f_{r 1}=34.5 \mathrm{~Hz}$ across nearly all $f_{m}$ components. Since these components are less related to gear mesh process, they need to be excluded for the focus on gear transmission monitoring. Nevertheless, MSB magnitudes exhibit many distinctive peaks at the mesh and shaft frequencies that are associated with gear transmission dynamics.

To locate the gear components more accurately, a contour plot of Figure 5(b) is presented in Figure 6. It shows that all the acoustic components can be separable as the MSB-coh peaks represented by dots are distinguishable in the $f_{m}-f_{r}$ bifrequency plane. For all gear transmissions of interest, several MSB peaks can be observable at the low speed mesh frequencies such as $f_{m 2}=200.01$ $\mathrm{Hz}$ and its three harmonics at $400.02 \mathrm{~Hz}, 600.03 \mathrm{~Hz}$ and $800.04 \mathrm{~Hz}$. Particularly, the peaks at bifrequencies of (400.02, 3.39) $\mathrm{Hz}$ and $(400.02,6.78) \mathrm{Hz}$ indicate a clear coupling between $f_{m 2}$ and $f_{r 3}$ as they are all associated with the low-speed stage of GB1. For the gear rotating at $f_{r 2}$, there is a peak at $(400.01,15.4) \mathrm{Hz}$. These coupling relations can be easily understood by the gear arrangement shown in Figure 2. Moreover, these observable peaks prove that the acoustic signals contain rich information about gear dynamics. For this baseline operation, it indicates that these gears have inherent imperfection such as pitch error and eccentricity. In the same way it can also identify the coupling peaks at bifrequencies $\left(f_{m 1}, f_{r 1}\right)$ and $\left(f_{m 1}, 2 f_{r 1}\right),\left(f_{m 1}, 1 f_{r 2}\right)$ and $\left(f_{m 1}, 2 f_{r 2}\right)$ which represent the dynamic characteristics of the inherent imperfect gears for the high-speed stage of GB1.

Based on the characteristics of the peaks and the fact that these peaks will change and more peaks relating to gear transmissions will emerge as gear engagements become poor with operation service time, a gear-monitoring indicator is defined by combining the MSB magnitude peaks at gear mesh frequency and its first few harmonics according to:

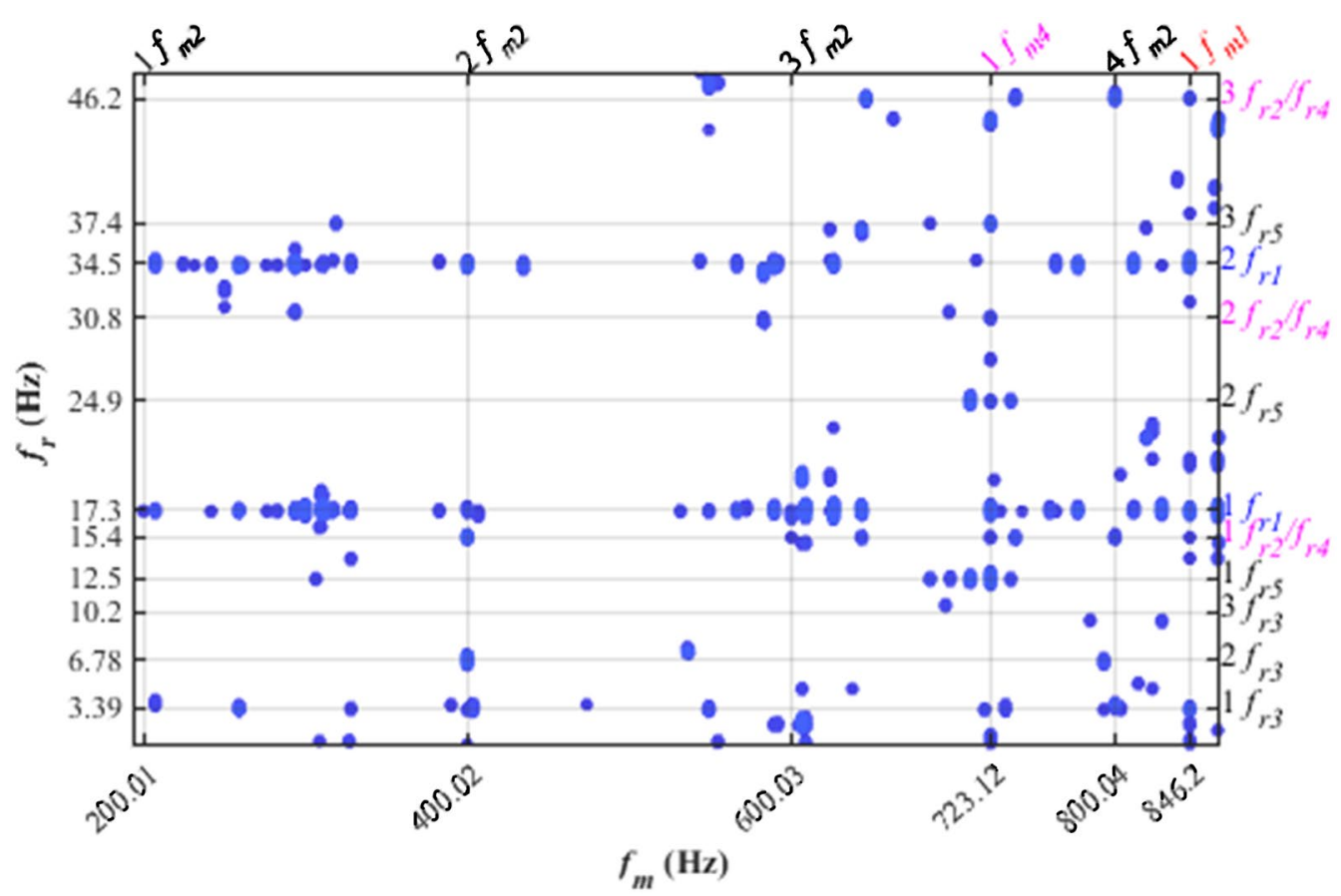

Figure 6 Baseline MSB coherence of Mic.1 at $325 \mathrm{~h}$ 


$$
\begin{aligned}
\operatorname{GMSB}\left(f_{m}\right)= & \sum_{n=1}^{3} \sum_{k=1}^{3}\left|B_{M S}\left(n f_{m}, k f_{r p}\right)\right| \\
& /\left|B_{M S}\left(n f_{m}, k f_{r p}\right)\right| \\
& +\sum_{n=1}^{3} \sum_{k=1}^{3}\left|B_{M S}\left(n f_{m}, k f_{r g}\right)\right| \\
& / \overline{\left|B_{M S}\left(n f_{m}, k f_{r g}\right)\right|},
\end{aligned}
$$

where $f_{r p}$ is the frequency of pinion rotation, $f_{r g}$ is the frequency of gear, and $k$ and $n$ are the harmonic orders for the shaft and mesh frequencies respectively. This monitoring feature will capture the information with respect to uniform tooth defects, which often leads to increases in the amplitude of tooth meshing harmonics, and the amplitudes of meshing harmonic sidebands $[8,32]$. And unlike previous features as demonstrated in Refs. [30, 31] using the motor current and vibration signals respectively, the proposed $G M S B\left(f_{m}\right)$ is the average of a number of noise purified MSB peaks and thus, will be more stable and accurate for tracking the conditions of progressive gear deteriorations. In addition, the normalization factor $\overline{\left|B_{M S}\left(n f_{m}, k f_{r x}\right)\right|}$ is calculated as the mean of a number of MSB peaks in baseline operation (the first 20 $h$ of the testing). This ensures that MSB peaks with different amplitudes still produce the equal contributions when applying Eq. (6), and thus changes in all peaks can be highlighted.

\subsection{Acoustic Trends and Diagnostics}

In order to trace the gear health condition with operating time for the two gearboxes respectively, $\operatorname{GMSB}\left(f_{m 1}, t\right)$ $\operatorname{GMSB}\left(f_{m 2}, t\right), \operatorname{GMSB}\left(f_{m 3}, t\right), \operatorname{GMSB}\left(f_{m 4}, t\right)$ for the four sets of gears were calculated using the acoustic signals at different operating time $t$ and then further combined according to:

$$
\operatorname{GMSB}_{G B 1}(t)=\left[\operatorname{GMSB}\left(f_{m 1}, t\right)+\operatorname{GMSB}\left(f_{m 2}, t\right)\right] / 2,
$$

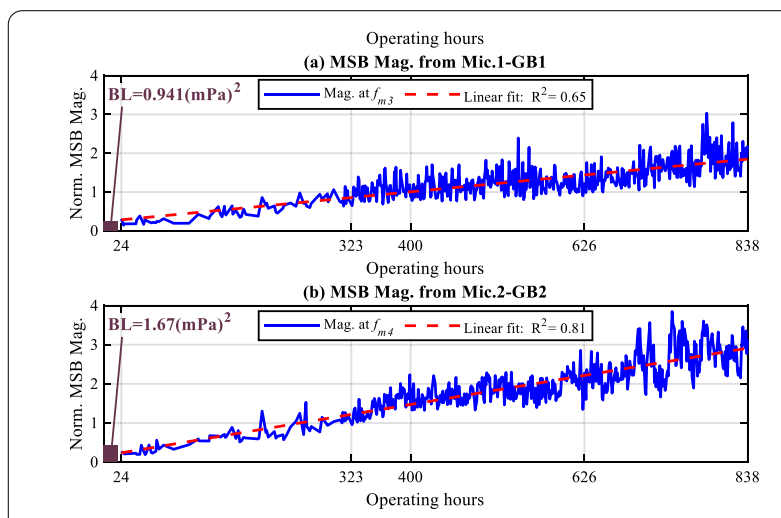

Figure 7 Evolution of acoustic MSB magnitude peaks with operating time: (a) GB1, (b) GB2

$$
\operatorname{GMSB}_{G B 2}(t)=\left[\operatorname{GMSB}\left(f_{m 3}, t\right)+\operatorname{GMSB}\left(f_{m 4}, t\right)\right] / 2,
$$

Figure 7 presents two GMSB trends for the test course. It can be seen that both gearboxes endured gradual deteriorations with operating time, which is well consistent with theoretical predictions of inevitable operation deteriorations. Especially, at the late operating time just before $838 \mathrm{~h}, \mathrm{~GB} 2$ shows an approximately threefold increase in GMSB values with higher fluctuations. This indicates the gearbox may have a significant deterioration, thereby, the test course was stopped at 838 $\mathrm{h}$ to avoid any damages to the test facilities by further operation.

A further study of the two trends has observed that GB2 exhibits a higher increase in GMSB values, which can be explained by the higher baseline peaks $\left(\mathrm{BL} 2=1.67(\mathrm{mPa})^{2}\right.$ while BL1 $\left.=0.941(\mathrm{mPa})^{2}\right)$. This means that GB2 probably has higher gear errors which results in higher deteriorating rates. This smaller baseline error also explains that GB1 exhibits more nonlinearity, and remains relatively stable for a longer period between $323 \mathrm{~h}$ to $626 \mathrm{~h}$, indicating that GB1 is more load resistance and has longer lifetime.

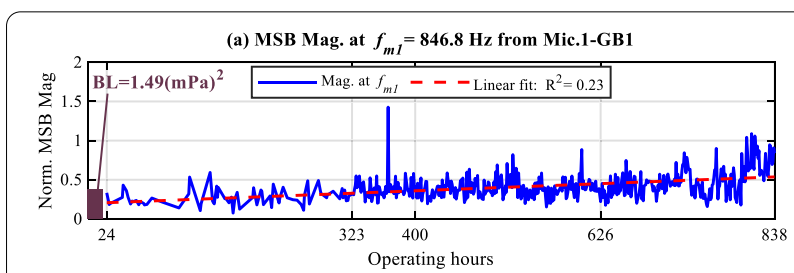

(b) MSB Mag. at $f_{m 2}=200.2 \mathrm{~Hz}$ from Mic.1-GB1

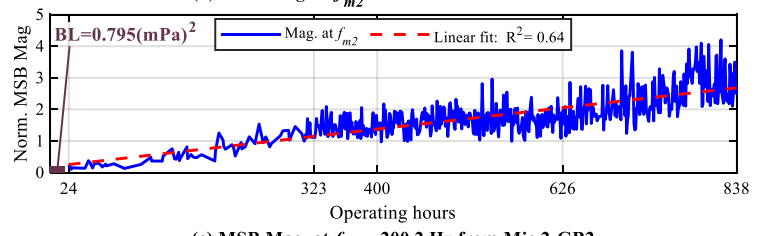

(c) MSB Mag. at $f_{m 3}=200.2 \mathrm{~Hz}$ from Mic.2-GB2

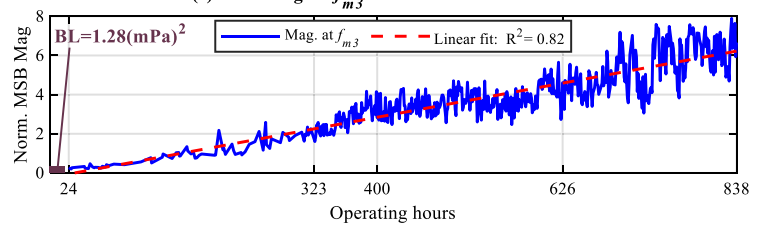

(d) MSB Mag. at $f_{m 4}=723.7 \mathrm{~Hz}$ from Mic.2-GB2

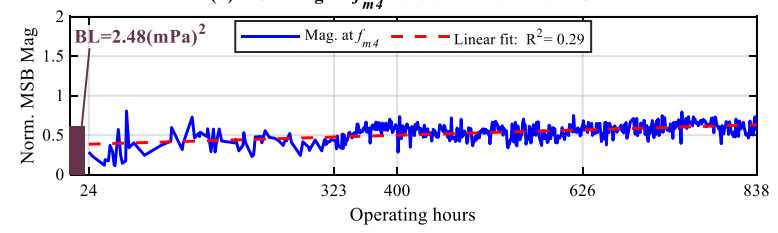

Figure 8 Evolution of acoustic MSB magnitude peaks for four mesh frequencies 


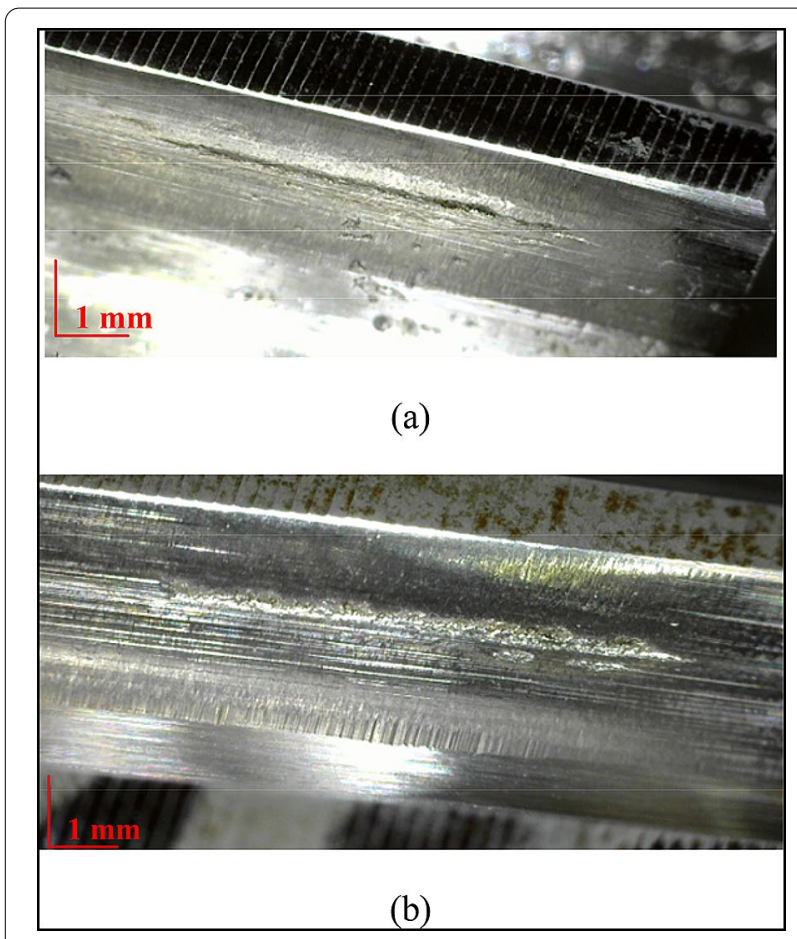

Figure 9 Illustration of tooth wear on the tooth surfaces of low speed gears (a) $Z_{r 3}$ and (b) $Z_{r 4}$

To diagnose the source of the deterioration, GMSB trends for each mesh gear pair are presented in Figure 8. It can be clearly seen that the low speed stages for both gearboxes show significant deterioration as both trends show steady increase with time as shown in Figure 8(b) and (c). In particular, GB2 shows an approximated sixfold increase while GB1 shows an approximated threefold increase, indicating that the gears of GB2 at the lowspeed stage is more severely deteriorated.

However, the gears at high-speed stages for both gearboxes show little changes during the operating course, as illustrated in Figure 8(a) and (d). This indicates that the engagement conditions of the high-speed gears are much less affected during the testing. By opening and inspecting both gearboxes after the tests were completed, it was found that the tooth surfaces of the low speed gears have clear wear and incipient pitting marks as illustrated by the images of Figure 9. However, the tooth surfaces of high-speed gears show minimal deterioration. This means that the low speed gears showed greater degradation as they are more prone to surface wear since the elastohydrodynamic (EHD) films are thinner under higher load and lower speed. Moreover, this inspection result fully confirms the monitoring and diagnostics achieved by the acoustic monitoring.

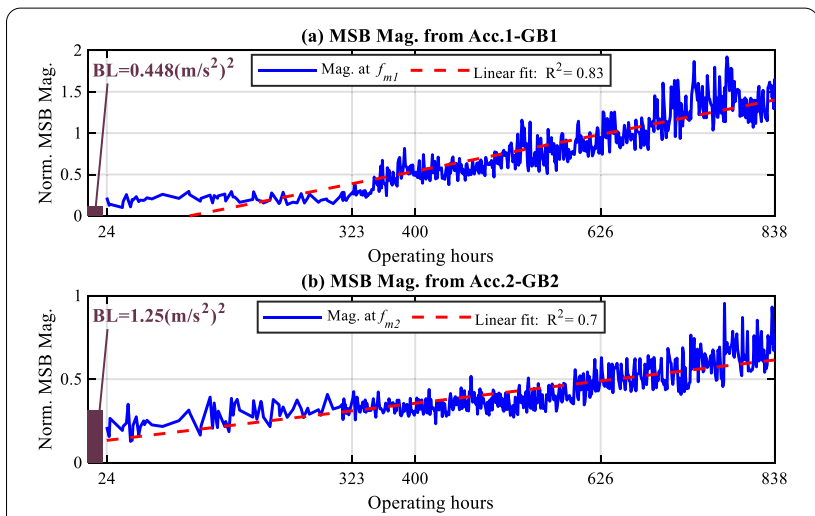

Figure 10 Evolution of vibration MSB magnitude peaks with operating time: (a) GB1, (b) GB2

Based on the analysis made above, acoustic signals perform superbly in tracing the gradual wears of gears. In particular, the monitoring features combined by MSB peaks allow the gradual changes in gearbox deterioration to be highlighted.

\subsection{Benchmark with Vibration Trends}

By utilizing the same technique, GMSB monitoring trends for the vibration measures were obtained and shown in Figure 10. Similarly, the trends for both gearboxes exhibit a gradual increase with operating time. This shows that acoustic monitoring is consistent with vibration one. However, as can be seen from the general trend, they exhibit more nonlinearities with time. This is probably because that vibration signals are from a localized area of the gearbox housing, which are more prone to the influence of nonlinear transmission paths. Moreover, this localized vibration perception is probably less reflective of the global vibration responses from different vibration excitations. Comparatively, the sound superposition radiated from the vibrations of different gearbox parts and perceived by the acoustic microphones can better globally represent the overall dynamics of the gearbox condition.

This difference in sensing the dynamics of gearbox also causes the vibrations to have lower sensitivity to changes in gear deteriorations. As can be seen from Figure 10, there are only about tripling and doubling increase with respect to GB1 and GB2, which is far less sensitive to the change in gradual wear process. Furthermore, GB2 shows a lower increase or less variation even though the baseline vibration is higher, which is inconsistent with the dynamic effects that higher vibrations would cause a faster wear progression to the gears. 


\section{Conclusions}

This paper introduces an acoustics based condition monitoring approach to the gear wear. It uses modulation signal bispectrum to suppress random noise and non-modulation content in the airborne signals that were acquired remotely, thereby results in accurate MSB peaks to indicate the gear transmission conditions. Based on the theoretical and experimental analysis, main conclusions are drawn as follows:

(1) Acoustic signals could contain comprehensive information about gear dynamics. The MSB magnitude peaks at the first three harmonic components of gear mesh and rotation components are generally sufficient to demonstrate the gradual deterioration of gear transmission systems with operating time.

(2) The gear monitoring indicator of GMSB defined by combining the MSB magnitude peaks at gear mesh frequency and its first few harmonics has proved to be consistent with the theoretical and experimental results. The gears of GB2 at the low-speed stage endured more severe deterioration with an approximately sixfold increase in GMSB values.

(3) Combining the bi-spectral peaks with baseline normalization using acoustic signals provides more sensitive (about 2 times higher) and thus accurate monitoring trends and diagnostics, allowing the gradual deterioration process and gear wear location to be represented reliably when compared with vibration based monitoring.

\section{Acknowledgements}

Not applicable.

\begin{abstract}
Authors' Contributions
$\mathrm{KL}$ carried out the study and drafted the manuscript. JG conducted signal processing and proofreading. HF carried out vibration signal based analysis. XS assisted in experimentation. BL conducted fault diagnosis part. FG was in charge of the methodology. All authors read and approved the final manuscript.
\end{abstract}

\section{Authors' Information \\ Kaibo Lu, born in 1984, is currently an associate professor at College of Mechanical and Vehicle Engineering, Taiyuan University of Technology, China. He received his PhD degree on Mechanical Engineering from Xi'an Jiaotong Uni- versity, China, in 2014. He has been an academic visitor at Centre for Efficiency and Performance Engineering (CEPE), University of Huddersfield, UK. His research interests include condition monitoring and fault diagnosis, and dynamics- based modelling and analysis.}

James Xi Gu, born in 1985, is a lecturer with the School of Engineering, University of Bolton, UK. He has a BEng from University of Manchester, UK, a MSc from Nottingham Trent University, UK and a PhD from Manchester Metropolitan University, UK. His research interests focus on smart engineering technology, condition monitoring and signal processing.

Hongwei Fan, born in 1984, is an associate professor at College of Mechanical Engineering, Xi'an University of Science and Technology, China. His research interests include rotor-bearing dynamics, and intelligent monitoring techniques for mining machinery.

Xiuquan Sun, born in 1991, is currently is a PhD candidate at Taiyuan University of Technology, China, and also is a joint PhD candidate at University of Huddersfield. His research interests include gear dynamics analysis, gear tribology, condition monitoring and fault diagnosis of gear transmission system.

Bing Li, born in 1971, is an associate professor of mechanical engineering at the School of Industrial Automation and the director of Engineering Training Centre of Zhuhai of, Beijing Institute of technology, Zhuhai, China. His research interests include fault diagnosis, friction and lubrication, metal and polymer processing technology.

Fengshou Gu, born in 1957, is a professor in diagnostic engineering, working as the Head of Measurement and Data Analytics Research Group (MDARG) and the Deputy Director of the CEPE at the University of Huddersfield, UK. He had his BSc, MSc from Taiyuan University of Technology, China and PhD from University of Manchester, UK. His research interests include machine dynamics, tribology dynamics, advanced signal processing, measurement system and sensor development, artificial intelligence and condition monitoring and diagnostics.

\section{Funding}

Supported by Shaanxi Key Laboratory of Mine Electromechanical Equipment Intelligent Monitoring, Xi'an University of Science and Technology (Grant No. SKL-MEEIM201904), and National Natural Science Foundation of China (Grant Nos. 51805352, 51605380)

\section{Competing Interests}

The authors declare that they have no competing interests.

\section{Author Details}

${ }^{1}$ College of Mechanical and Vehicle Engineering, Taiyuan University of Technology, Taiyuan 030024, China. ${ }^{2}$ School of Engineering, University of Bolton, Bolton BL3 5AB, UK. ${ }^{3}$ College of Mechanical Engineering, Xi'an University of Science and Technology, Xi'an 710054, China. ${ }^{4}$ Centre for Efficiency and Performance Engineering, University of Huddersfield, Huddersfield HD1 3DH, UK. ${ }^{5}$ School of Industrial Automation, Beijing Institute of Technology, Zhuhai 519088, China.

Received: 2 June 2020 Revised: 13 April 2021 Accepted: 16 August 2021 Published online: 30 August 2021

\section{References}

[1] R B Randall. Vibration-based condition monitoring: industrial, aerospace and automotive applications. Wiley, 2011.

[2] Y Lei, J Lin, M Zuo, et al. Condition monitoring and fault diagnosis of planetary gearboxes: A review. Measurement, 2014, 48: 292-305.

[3] L Wang, Y Shao. Crack fault classification for planetary gearbox based on feature selection technique and K-means clustering method. Chinese Journal of Mechanical Engineering, 2018, 31: 4.

[4] TWang, Q Han, F Chu, et al. Vibration based condition monitoring and fault diagnosis of wind turbine planetary gearbox: A review. Mechanical Systems and Signal Processing, 2019, 126: 662-685

[5] R Teti, K Jemielniak, G O'Donnell, et al. Advanced monitoring of machining operations. CIRP Annals-Manufacturing Technology, 2010, 59: 717-739.

[6] K Lu, Z Lian, F Gu, et al. Model-based chatter stability prediction and detection for the turning of a flexible workpiece. Mechanical Systems and Signal Processing, 2018, 100: 814-826.

[7] A Mohanty, C Kar. Fault detection in a multistage gearbox by demodulation of motor current waveform. IEEE Transactions on Industrial Electronics, 2006, 53(4): 1285-1297.

[8] C Hu, W A Smith, R B Randall, et al. Development of a gear vibration indicator and its application in gear wear monitoring. Mechanical Systems and Signal Processing, 2016, 76-77: 319-336.

[9] B S Payne, A D Ball, F Gu, et al. A head-to-head assessment of the relative fault detection and diagnosis capabilities of conventional vibration 
and airborne acoustic monitoring. Proceedings of the 13th International Congress on Condition Monitoring and Diagnostic Engineering Management (COMADEM 2000), Texas, USA, 2000.

[10] L Barelli, G Bidini, C Buratti, et al. Diagnosis of internal combustion engine through vibration and acoustic pressure non-intrusive measurements. Applied Thermal Engineering, 2009, 29: 1707-1713.

[11] F Gu, W Li, A Ball, et al. The condition monitoring of diesel engines using acoustic measurements part 1: Acoustic characteristics of the engine and representation of the acoustic signals. SAE Technical Paper 2000-01-0730, 2000, https://doi.org/10.4271/2000-01-0730.

[12] A Ball, F Gu, W Li. The condition monitoring of diesel engines using acoustic measurements part 2: Fault detection and diagnosis. SAE Technical Paper 2000-01-0368, 2000, https://doi.org/10.4271/2000-01-0368.

[13] W Li, R M Parkin, J Coy, et al. Acoustic based condition monitoring of a diesel engine using self-organising map networks. Applied Acoustics, 2002, 63(7): 699-711.

[14] A Albarbar, F Gu, A Ball. Diesel engine fuel injection monitoring using acoustic measurements and independent component analysis. Measurement, 2010, 43: 1376-7386.

[15] J Jiang, F Gu, R Gennish, et al. Monitoring of diesel engine combustions based on the acoustic source characterisation of the exhaust system. Mechanical Systems and Signal Processing, 2008, 22(6): 1465-1480.

[16] J Zhou, W Sun. Vibration and noise radiation characteristics of gear transmission system. Journal of Low Frequency Noise, Vibration and Active Control, 2014, 33: 485-502.

[17] T Delio, J Tlusty, S Smith. Use of audio signals for chatter detection and control. Journal of Manufacturing Science and Engineering, 1992, 114 . 146-157.

[18] N Seemuang, T McLeay, T Slatter. Using spindle noise to monitor tool wear in a turning process. International Journal of Advanced Manufacturing Technology, 2016, 86: 2781-2790.

[19] C H Lauro, L C Brandao, D Baldo, et al. Monitoring and processing signal applied in machining processes - A review. Measurement, 2014, 58: 73-86.

[20] U Greb, M G Rusbridge. The interpretation of the bispectrum and bicoherence for non-linear interactions of continuous spectra. Plasma Physics and Controlled Fusion, 1988, 30(5): 537-549.

[21] F Gu, Y Shao, N Hu, et al. Electrical motor current signal analysis using a modified bispectrum for fault diagnosis of downstream mechanical equipment. Mechanical Systems and Signal Processing, 2011, 25(1): 360-372.

[22] F Gu, TWang, A Alwodai, et al. A new method of accurate broken rotor bar diagnosis based on modulation signal bispectrum analysis of motor current signals. Mechanical Systems and Signal Processing, 2015, 50-51: 400-413.

[23] X Liang, Z Liu, J Pan, et al. Spur gear tooth pitting propagation assessment using model-based analysis. Chinese Journal of Mechanical Engineering, 2017, 30(6): 1369-1382.

[24] Y Li, K Ding, G He, et al. Vibration mechanisms of spur gear pair in healthy and fault states. Mechanical Systems and Signal Processing, 2016, 81: 183-201.

[25] F K Choy, V Polyshchuk, J J Zakrajsek, et al. Analysis of the effects of surface pitting and wear on the vibration of a gear transmission system. Tribology International, 1996, 29: 77-83.

[26] D Yassine, H Ahmed, W Lassaad, et al. Effects of gear mesh fluctuation and defaults on the dynamic behavior of two-stage straight bevel system. Mechanism and Machine Theory, 2014, 82: 71-86.

[27] H Jiang, Y Shao, C K Mechefske. Dynamic characteristics of helical gears under sliding friction with spalling defect. Engineering Failure Analysis, 2014, 39: 92-107.

[28] H Ma, J Zeng, R Feng, et al. An improved analytical method for mesh stiffness calculation of spur gears with tip relief. Mechanism and Machine Theory, 2016, 98: 64-80.

[29] Z Chen, TWang, F Gu, et al. Gear transmission fault diagnosis based on the bispectrum analysis of induction motor current signatures. Journal of Mechanical Engineering, 2012, 48(21): 84-90. (in Chinese)

[30] R Zhang, F Gu, H Mansaf, et al. Gear wear monitoring by modulation signal bispectrum based on motor current signal analysis. Mechanical Systems and Signal Processing, 2017, 94(15): 202-213.

[31] R Zhang, X Gu, F Gu, et al. Gear wear process monitoring using a sideband estimator based on modulation signal bispectrum. Applied Sciences, 2017, 7(3): 274.

[32] Y Lei, N Li, L Guo, et al. Machinery health prognostics: A systematic review from data acquisition to RUL prediction. Mechanical Systems and Signal Processing, 2018, 104: 799-834.

\section{Submit your manuscript to a SpringerOpen ${ }^{\odot}$ journal and benefit from:}

- Convenient online submission

- Rigorous peer review

- Open access: articles freely available online

- High visibility within the field

- Retaining the copyright to your article

Submit your next manuscript at $\boldsymbol{\nabla}$ springeropen.com 